\title{
Studies on the Metabolism of the Chrysophyceae
}

\section{COMPARATIVE STRUCTURAL INVESTIGATIONS ON LEUCOSIN (CHRYSOLAMINARIN) SEPARATED FROM DIATOMS AND LAMINARIN FROM THE BROWN ALGAE}

\author{
By ANNE BEATTIE, E. L. HIRST and ELIZABETH PERCIVAL \\ Chemistry Department, University of Edinburgh
}

(Received 17 October 1960)

The Chrysophyceae comprise a large number of plankton and benthic algae of fresh and sea water; many, if not all, of the diatoms belonging to this Order synthesize an intracellular polysaccharide, described by the phycologists as leucosin. This carbohydrate occurs dissolved in the cell vacuole, the refractive index of the latter being proportional to its leucosin content. Pringsheim (1952) is, however, of the opinion that the term leucosin has been applied to very different substances; indeed this polysaccharide has been variously described as a polyfructose resembling inulin and a polyglucose similar to laminarin. Stosch (1951) separated an insoluble form of leucosin from freshwater diatoms and both an insoluble and soluble form of this polysaccharide from marine diatoms (H. A. von Stosch, unpublished communication to the Third International Seaweed Symposium, Galway, 1958). This author considers that, in view of its solubilities and the chromatographic behaviour of the derived mono- and di-saccharides, leucosin is closely related to the soluble and insoluble forms of laminarin, essentially linear $\beta-1: 3^{\prime}$-linked glucans synthesized by various species of the brown marine algae (Phaeophyceae). A polysaccharide termed leucosin has also been separated from the flagellated protozoon Ochromonas malhamensis. Archibald, Manners \& Ryley (1958) have established that this material resembles laminarin in that it, too, consists of $\beta-1: 3^{\prime}$-linked glucose units, with a small proportion of other linkages also present.

The difficulty of isolating sufficient leucosin from the Chrysophyceae has precluded systematic structural investigations on this polysaccharide. Quillet (1955) found that leucosin extracted from Hydrurus foetidus had $[\alpha]_{D}-6^{\circ}$, gave no stain with iodine and chromatographic examination of an acid hydrolysate revealed that glucose was the only sugar present. A paper chromatogram of a partial acid hydrolysate showed six evenly spaced spots together with several spots near the starting line, from which Quillet concluded that the polysaccharide contained at least eight anhydroglucose units. In view of the ambiguity of the term leucosin this author suggests that a better name for this material would be chrysolaminarin. The recent development of new techniques has made possible structural investigations of polysaccharides on milligram quantities and this paper describes the results obtained from $0.5 \mathrm{~g}$. of crystalline (in. soluble) leucosin, hereinafter called chrysolamin. arin. This material was separated from a mixture of diatoms belonging to the Chrysophyceae, and was placed at the disposal of the authors through the generosity of Professor H. A. von Stosch. In view of the limited supply of polysaccharide, and of its possible identity with the laminarin isolated from the brown seaweeds, it appeared advisable to carry out parallel experiments on insoluble laminarin isolated from Laminaria cloustoni. The similarities and differences between the two polysaccharides are discussed.

\section{MATERIALS AND METHODS}

\section{Analytical methods}

Chromatography. (a) Qualitative. Descending paper chromatograms were prepared at room temperature with Whatman no. 1 paper and butanol-pyridine-benzenewater (5:3:1:3, by vol.) (A), butan-2-one (ethyl methyl ketone)-acetic acid-water saturated with boric acid (9:1:1, by vol.) (B), ethyl acetate-pyridine-water $(10: 4: 3)$ (C), ethyl acetate-acetic acid-formic acid-water (18:3:1:4) (D), butanol-ethanol-water $(4: 1: 5)$ (E), butan-2-onewater (10:1) (F) and butanol-pyridine-water (10:3:3) (G) as solvents. Aniline oxalate (i), silver nitrate-sodium hydroxide (ii) [for details of (i) and (ii) see Bell (1955)], periodate-benzidine (iii) (Cifonelli \& Smith, 1954), bromocresol green (iv) $(0.1 \%$ soln. in $95 \%$ ethanol made alkaline with sodium hydroxide) were used as spray reagents. The rate of movement of sugars is expressed relative to tetra- $O$. methylglucose $\left(R_{G}\right.$ values) or to glucose ( $R_{\mathrm{Gl}}$ values).

(b) Quantitative. The methylated sugars were estimated by the method of Schaefer \& Van Cleve (1956).

Electrophoresis. Separation on Whatman no. 1 paper was effected in an apparatus described by Foster (1952) at $750 \mathrm{v}$ and $10 \mathrm{~mA} .0 \cdot 1 \mathrm{M}$-Borate buffer, $\mathrm{pH} 10 \cdot 0$, was used.

Acid hydrolysis. (a) Complete acid hydrolysis was carried out by heating the polysaccharide with $\mathrm{N}$-sulphuric acid $\left(1.5 \%\right.$ soln., w/v) at $100^{\circ}$ until the rotation was constant $(6 \mathrm{hr}$.). Reducing sugar was determined with the Somogyi (1952) reagent calibrated against glucose.

(b) Partial acid hydrolysis was effected by heating with $0.1 \mathrm{~s}$-sulphuric acid $\left(0.5 \%\right.$ soln., w/v) for $1 \mathrm{hr}$. at $100^{\circ}$. 
(c) Chrysolaminaritol and laminaritol were each hydrolysed with $\mathrm{N}$-sulphuric acid for $5 \mathrm{hr}$. at $100^{\circ}$.

(d) The methylated polysaccharides (see below) were hydrolysed with $90 \%$ formic acid in a sealed tube under carbon dioxide for $4 \mathrm{hr}$. in a boiling-water bath, then diluted with water (5 vol.) and heated for a further $1 \mathrm{hr}$. The mixture was filtered from Filter-Cel and glass and the filtrate deionized by passage through a column of mixedbed resin [Amberlite IR-120(H) and IR-4B(OH)]. The column was washed both with water and with methanol and the combined eluents, after filtration, were evaporated to dryness.

Periodate oxidation. The consumption of periodate and production of formaldehyde were determined by the methods of Aspinall \& Ferrier (1957) and Hough, Powell \& Woods (1956) respectively. Formaldehyde was also determined with the chromotropic acid reagent by the methods of MacFadyen, Watkins \& Anderson (1945), as modified by Parrish (1959) and Hough \& Perry (1956). The degree of polymerization was measured from the formaldehyde released on oxidation with periodate. All the experiments with periodate were carried out in duplicate.

Moisture. The loss of weight on drying the chrysolaminarin at $60^{\circ} / 15 \mathrm{~mm}$. mercury over phosphoric oxide for $24 \mathrm{hr}$. was used to calculate the moisture content.

\section{RESULTS}

\section{Isolation and purification of the chrysolaminarin}

The material used in these experiments was isolated by Professor H. A. von Stosch by the following procedure.

The water bloom on a small pond in the Botanical Gardens of Darmstadt was collected in early spring. Microscopic examination showed that it contained the following species of diatoms: Nitzschia sigmoidea, Cymatopleura solea, Pinnularia sp. and Melosira varians. The mixed culture was extracted with aq. $50 \%$ ethanol or hot water, and the extracts were deproteinized by treatment with basic lead acetate, followed by precipitation of the lead with hydrogen sulphide. In some experiments the chrysolaminarin was isolated by allowing it to deposit from the concentrated protein-free solution on standing. In other cases it was crystallized from diacetin and the product washed with ethanol and and ether. Twelve different collections of bloom were made and the crystalline extracts were combined and recrystallized twice from water. Yield was about $1 \cdot 0 \mathrm{~g}$.

\section{Characterization of chrysolaminarin}

The crystalline chrysolaminarin has m.p. $273^{\circ}$, $[\alpha]_{\mathrm{D}}^{18}-6^{\circ}$ in water $(c, 2.0)$ (moisture, $11 \%$ ). The dried material has the same crystalline appearance under the microscope and the same m.p. as the initial material. An aqueous solution of the latter gave no colour with iodine solution.

Glucose content. Hydrolysis of the dried chrysolaminarin (50.4 mg.), followed by determination of the reducing value, indicated that the polysaccharide contained $99.5 \%$ of glucose, assuming it to be a polymer of glucose. The hydrolysate had $[\alpha]_{\mathrm{D}}^{18}+52 \cdot 8^{\circ}$ in water $(c, 0 \cdot 5)$ and after neutralization with barium carbonate and concentration the resulting syrup crystallized. The crystals had m.p. and mixed m.p. with glucose hydrate $83^{\circ}$. That glucose was the only sugar liberated by acid hydrolysis was shown by incubating a sample of the hydrolysate with notatin, glucose oxidase, at $37^{\circ}$ for $6 \mathrm{hr}$. The resulting solution was non-reducing and chromatographic examination (solvent $\mathrm{D}$, spray iv) showed that the single spot given by the original hydrolysate (solvents $\mathrm{A}$ and $\mathrm{C}$, spray i) had been replaced by a spot corresponding to gluconic acid. A hydrolysate derived from laminarin (50 mg.) showed on chromatographic examination (solvent $B$, spray iii) in addition to glucose a spot identical in speed and colour with mannitol run as a control. This spot was absent from a comparable chromatogram of the hydrolysate from chrysolaminarin.

Partial hydrolysis of laminarin gave a hydrolysate which on chromatographic examination (solvent G, $132 \mathrm{hr}$. development) revealed spots with $R_{\mathrm{Gl}} 0.71,0.56,0.43,0.35,0.26,0.21,0 \cdot 15,0 \cdot 13$, 0.08. Laminari-biose and -triose run as controls had $R_{\mathrm{G} 1} 0.71$ and 0.43 respectively.

Borohydride reduction. Each of the dried polysaccharides, chrysolaminarin (132.9 mg.) and laminarin $(240 \mathrm{mg}$.$) , were reduced with potassium$ borohydride $(0.1 \mathrm{~g}$.$) in water (10 \mathrm{ml}$.) with stirring for $48 \mathrm{hr}$. at room temperature. The mixtures were neutralized ( $\mathrm{pH} \mathrm{7,} \mathrm{acetic} \mathrm{acid)} \mathrm{and} \mathrm{the} \mathrm{polysac-}$ charides precipitated with ethanol. They were purified by twice dissolving in water and precipitating with ethanol. Chrysolaminaritol (91\% yield) and laminaritol (95\%) were isolated with $92 \%$ and $93 \%$ glucose contents respectively. Insufficient material was available to determine the reducing power of the chrysolaminaritol, but the laminaritol $(88 \mathrm{mg}$.$) had no reducing power, and$ since the reduction of the two polysaccharides was carried out under identical conditions it may be assumed that the reduction of the chrysolaminarin was complete. Reduction with borohydride converts all the reducing glucose residues in laminarin and chrysolaminarin into sorbitol units. The mannitol units are unattacked. Hydrolysates of the two polysaccharide alcohols (30 mg.) were examined by paper chromatography (solvent $B$, spray iii). From laminaritol spots, corresponding to glucose, mannitol and sorbitol were obtained, and from chrysolaminaritol, glucose and sorbitol. Visual examination indicated a relatively higher concentration of sorbitol from chrysolaminaritol.

Methylation of chrysolaminarin. The dried polysaccharide (85 mg.) was mixed with Filter-Cel 
(200 mg.) and methylated in liquid ammonia (about $3 \mathrm{ml}$.) under less than atmospheric pressure in the micro-apparatus devised by Isbell, Frush, Bruchner, Kowkabany \& Wampler (1957). Potassium (in portions of about $3 \mathrm{mg}$.) was added until the mixture became permanently blue ( $5 \mathrm{hr}$.). After complete removal of the ammonia, methyl iodide $(2 \mathrm{ml}$.) was introduced into the reaction vessel and the mixture refluxed with stirring for $18 \mathrm{hr}$. Then the methyl iodide was withdrawn from the reaction vessel and the methylation repeated five times before isolation of the polysaccharide. The following minor modifications were introduced into the procedure: (1) More efficient condensation of ammonia was achieved when acetone replaced the chlorobenzene in the cold finger. (2) A flat base to the reaction vessel lessened the possibility of the magnetic stirrer sticking. (3) The most satisfactory grease for the glass stopcocks was found to be paraffin and rubber lubricant (British Drug Houses Ltd.). (4) An additional stopcock in a tube at right angles to the side arm from which the potassium was introduced permitted re-evacuation of this arm whenever a fresh supply of potassium was introduced and thus prevented the slight loss of vacuum in the main apparatus. (5) It was found that a hair-drier was suitable for defrosting the stopcock used for the potassium addition, and also for heating the various parts of the glassware to remove completely both the ammonia and the methyl iodide. (6) To ensure easy removal of the cold finger it was found to be advantageous to renew the dry acetone in the outer part of the cold finger after each methylation.

The methoxyl content of the final product was not determined because it proved impossible to free the methylated polymer from contaminating grease.

Examination of the methylated hydrolysates. The partly crystalline solid (70 mg.) obtained on hydrolysis of the methylated chrysolaminarin was examined on a paper chromatogram (solvents $\mathbf{E}$ and F, spray i) and by electrophoresis. Spots with speeds identical with 2:3:4:6-tetra-, 2:4:6-tri- and 2:4-di-O-methylglucoses were obtained. No trace of 4:6-di- $O$-methylglucose was revealed on ionophoresis (2:4-di-O-methyl- and 4:6-di-O-methylglucose have $M_{\Theta} 0.09$ and 0.20 respectively; Mackie \& Percival, 1959). Determination of the relative molar proportions of tetra- to tri- to di-O. methylglucose (Schaefer \& Van Cleve, 1956) gave $1: 10 \cdot 5: 0 \cdot 46$. Some of the crystals were removed from the hydrolysate and freed from adhering syrup. They had m.p. and mixed m.p. with 2:4:6tri-O-methylglucose $118^{\circ}$.

The chromatographic spot corresponding to 2:4:6-tri- $O$-methylglucose was elongated when developed in solvent $\mathrm{F}$ and it was suspected that a small proportion of 2:3:4-tri- $O$-methylglucose might be present. The methylated sugars (14.45 mg.) were separated on chromatography paper (solvent E). The tetra-, tri- and di-O-methyl sugars were located by means of guide strips. The areas of paper containing the different sugars were cut out and the latter was eluted with water. The quantity of 2:3:4-tri- $O$-methylglucose in the tri- $O$-methyl fraction could not be determined by straightforward oxidation with periodate and determination of the quantity of formaldehyde released since steric factors prevent complete liberation of the latter. Prior reduction to the straight-chain alcohol removes this effect and leads to a quantitative yield of formaldehyde.

The tri- $O$-methyl sugars (5.0 mg.; $9.5 \mathrm{mg}$.) dissolved in water $(2 \mathrm{ml}$.) were reduced with potassium borohydride ( $8 \mathrm{mg}$.). After $2 \mathrm{hr}$. the solutions were neutralized with sulphuric acid, $0.3 \mathrm{M}$-sodium periodate $(0.3 \mathrm{ml}$.) was added and the solutions were each made up to $3 \mathrm{ml}$. and set aside for $30 \mathrm{~min}$. (see Perry \& Jones, 1957). A sample (1 ml.) was removed from each and analysed for formaldehyde (MacFadyen et al. 1945; Parrish, 1959). 2:4:6-Tri-O-methylglucose (2 mg.), 2:4:6tri- $O$-methylglucitol (2 mg.) and the eluate from a similar area of chromatography paper were oxidized with $0.3 \mathrm{M}$-sodium periodate as for the reduced tri- $O$-methyl fraction, and the resulting solutions were analysed for formaldehyde. Formaldehyde (57.6 and $114 \mu \mathrm{g}$.) was liberated from the solutions $(3 \mathrm{ml}$.) derived from the methylated chrysolaminarin. In contrast no formaldehyde was released in the other three experiments.

Confirmation of the presence of 2:3:4-tri-Omethylglucose was obtained by chromatographic examination of the residual periodate oxidation solution derived from methylated chrysolaminarin, after destruction of the excess of periodate with ethylene glycol and deionization. A spot corresponding to 2:3:4-tri- $O$-methylxylose (spray i) was revealed. Reduction of 2:3:4-tri-O-methylglucose followed by periodate oxidation yields 2:3:4-tri-O. methylxylose.

The yield of formaldehyde from the tri- $O$. methyl fraction from methylated chrysolaminarin corresponds to 1 unit of 2:3:4-tri- $O$-methylglucose to every 11.0 units of tri- $O$-methyl sugar, that is approximately one 1:6'-linked glucose unit to every 11 1:3'-linked residues.

Methylation of laminarin. Laminarin (50 mg.) was methylated twice and then hydrolysed under the conditions used for the chrysolaminarin. Subsequent estimation of the methylated sugars gave tetra-:tri-:di-O-methylglucose $=1: 16: 0 \cdot 6$. Chromatographic separation of the respective sugars and reduction and periodate oxidation of the tri- $O$-methyl $(11.2 \mathrm{mg}$.) fraction released 
$101 \mu \mathrm{g}$. of formaldehyde. This corresponds to about $7 \%$ of $2: 3: 4$-tri- $O$-methylglucose and indicates that there is on an average approximately one 1:6'-linked glucose unit to 15 1:3'-linked units.

The presence of one part of di-O-methylglucose in about 25 and 19 parts of methylated sugars from chrysolaminarin and laminarin respectively, unless it is due to undermethylation or demethylation or both during hydrolysis, provides evidence for the presence of about one branch point in every molecule. The high proportion of tetra- $O$-methylglucose from both methylated polysaccharides indicates that it is unlikely that either undermethylation or demethylation occurred to any appreciable extent in the present experiments.

Oxidation with periodate. Dried laminarin (14.05 mg.), dried chrysolaminarin (18.59 mg.), laminaritol (19.54 mg.), chrysolaminaritol (16.66 mg.), glucose (15.4 mg.) and sorbitol (24.07 mg.) were each dissolved in $0.015 \mathrm{M}$-sodium periodate $(15 \mathrm{ml}$.) and stored in the dark at $2^{\circ}$. Samples $(1 \mathrm{ml}$.) were analysed for periodate consumption and for formaldehyde release (Hough, Powell \& Woods, 1956). After $48 \mathrm{hr}$. the consumption of periodate appeared to be complete for both polysaccharides. After oxidation for $96 \mathrm{hr}$. at $2^{\circ}$ the residual oxidation solutions were allowed to stand at room temperature and samples $(1 \mathrm{ml}$.) were analysed as before (see Table 1).

The relatively small quantity of periodate reduced by laminarin provided evidence of chains of 1:3'-linked glucose units (Anderson, Hirst, Manners \& Ross, 1958). In the present experiments the similarity in the quantity of periodate consumed by the two polysaccharides indicates a similarity in structure and a high proportion of 1:3'-linked glucose residues in chrysolaminarin, a conclusion which finds support in the presence of a high proportion of unattacked glucose in the hydrolysates of the oxopolysaccharide and in the relatively large quantity of 2:4:6-tri- $O$-methylglucose in the hydrolysate of the methylated polysaccharide. A partial acid hydrolysate of the oxopolysaccharide of chrysolaminarin revealed on chromatographic analysis spots with $R_{\mathrm{G} 1} 0.71,0.43,0.26$ and $0 \cdot 15$. The absence of spots with $R_{\mathrm{Gl}} 0.56$ (gentiobiose), 0.35 (possibly 3-O- $\beta$-gentiobiosylglucose or 1-O- $\beta$-laminaribiosylmannitol; Peat, Whelan \& Lawley, 1958), $0.21,0.13$ given by a hydrolysate of unoxidized laminarin may be explained as due to cleavage of all the $1: 6^{\prime}$-linked units in chrysolaminarin by the periodate and consequently the loss of all the $1: 6^{\prime}$-linked oligosaccharides.

The action of periodate on laminarin under various conditions is described in detail by Anderson et al. (1958), and these and other workers (Peat et al. 1958) consider that laminarin, like starch, is made up of two molecular species. In one of these the chains of $1: 3^{\prime}$-linked glucose units are terminated by reducing glucose residues (G-chains) and in the other these residues are replaced by mannitol units (M-chains) linked through $\mathrm{C}_{(1)}$ or $\mathrm{C}_{(6)}$. More recently Smith \& Unrau (1959) have provided evidence that each mannitol unit in the mannitolcontaining molecules is linked through $\mathrm{C}_{(1)}$ and $\mathrm{C}_{(2)}$ (or $\mathrm{C}_{(6)}$ and $\mathrm{C}_{(5)}$ ) to two glucose chains and that these molecules contain two 1:6'-linked glucose units, whereas the molecules devoid of mannitol contain only one $1: 6^{\prime}$-linkage.

When subjected to oxidation with periodate the residues in laminarin that are attacked are the $1: 6^{\prime}$-linked units and the residues at the nonreducing ends of the chains, each of which reduce

Table 1. Oxidation of the polysaccharides with periodate

Oxidation was carried out with $15 \mathrm{~mm}$-sodium periodate in the dark. After $96 \mathrm{hr}$. at $2^{\circ}$ the oxidation was continued at room temperature $\left(18^{\circ}\right)$. Other details are given in the text.

(a) $\mathrm{NaIO}_{4}$ consumed (mole/anhydroglucose unit)

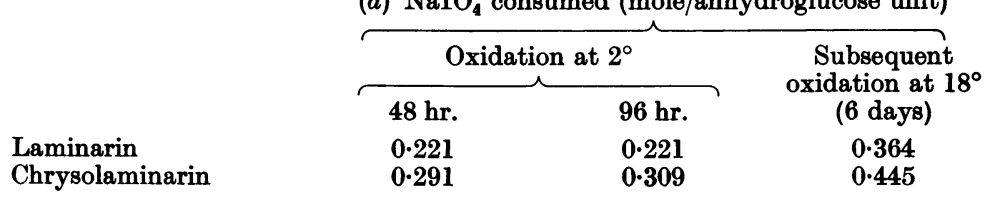

(b) Formaldehyde produced (mole/anhydroglucose unit)

\begin{tabular}{|c|c|c|c|c|c|c|c|}
\hline & \multicolumn{3}{|c|}{ Oxidation at $2^{\circ}$} & \multicolumn{4}{|c|}{ Subsequent oxidation at $18^{\circ}$} \\
\hline & $24 \mathrm{hr}$. & $48 \mathrm{hr}$. & $96 \mathrm{hr}$. & $96 \mathrm{hr}$. & 5 days & 6 days & 7 days \\
\hline Laminarin & 0.028 & 0.028 & 0.024 & 一 & 0.0468 & 0.0469 & 0.0469 \\
\hline Chrysolaminarin & 0.009 & $0 \cdot 008$ & - & - & 0.0462 & 0.0470 & $0 \cdot 0470$ \\
\hline Glucose & 0.009 & $0 \cdot 009$ & - & - & - & - & - \\
\hline Laminaritol & - & $0 \cdot 056$ & 0.056 & 0.066 & - & 0.069 & - \\
\hline Chrysolaminaritol & - & $0 \cdot 060$ & $0 \cdot 056$ & 0.077 & - & $0 \cdot 080$ & - \\
\hline Sorbitol & - & - & 0.77 & - & - & 0.91 & - \\
\hline
\end{tabular}


2 moles of periodate with the release of 1 mole of formic acid. At the same time the units at the other ends of the chains are also cleaved. If the reaction is carried out at $2^{\circ}$ the oxidation of these latter units in the G-chains is arrested after the reduction of 1 molecule of periodate owing to the formation of a formyl ester, which is hydrolysed very slowly at this temperature, and no formaldehyde is formed. The mannitol units at this end of the chains release 1 mole of formaldehyde (Anderson et al. 1958).

The release of 0.028 mole of formaldehyde for each anhydroglucose unit in laminarin corresponds to $2 \%$ of mannitol present in laminarin and is in agreement with the results of the earlier workers. The value recorded for chrysolaminarin (Table 1 ) is no greater than that for glucose, which is known to yield no formaldehyde under these conditions. This confirms the absence of M-chains in this polysaccharide.

At room temperature both $\mathbf{M}$-chains and Gchains release 1 mole of formaldehyde and from this release the average degree of polymerization of the polysaccharide may be calculated. In the present experiments (see Table 1) 1 mole of formaldehyde is released by every 21 anhydroglucose units in both polysaccharides.

The reduced chrysolaminarin should release 2 moles of formaldehyde from each of the newly formed sorbitol units and, assuming the absence of M-chains, chrysolaminaritol should give about $30 \%$ more formaldehyde than laminaritol. In fact, the yield of formaldehyde on oxidation of chrysolaminaritol at room temperature almost reaches the expected figure: 0.08 mole/anhydroglucose unit instead of the theoretical 0.090 mole for the complete absence of mannitol-ended chains. This yield is considerably higher than that from laminaritol and is further evidence that M-chains, if present in chrysolaminarin, must be very small in number. Comparable experiments with sorbitol show that $100 \%$ release of formaldehyde on oxidation with periodate is very difficult to attain and this may explain the slightly low yield from chrysolaminaritol. It will be observed from Table 1 that the yield of formaldehyde at $2^{\circ}$ in $96 \mathrm{hr}$. is low, but this can be explained by the observed slow release from sorbitol at this temperature.

Oxidation with excess of periodate at $\mathrm{pH} 8$ and $35^{\circ}$. Portions ( $1 \mathrm{ml}$.) of each of the polysaccharide solutions prepared as described in the previous section were withdrawn immediately after mixing, and phosphate buffer (6.8 ml., pH 8), together with excess of sodium periodate $(1 \mathrm{ml} ., 0.3 \mathrm{M})$, were added and the solutions diluted to $10 \mathrm{ml}$. with water. After incubation at $35^{\circ}$ the release of formaldehyde was determined on samples $(1 \mathrm{ml}$.
(Hough \& Perry, 1956) and found to be complete after $48 \mathrm{hr}$. The amounts of formaldehyde released (mole/anhydroglucose unit) were: laminarin, $\mathbf{0 . 6 0 0}$; laminaritol, 0.603; chrysolaminarin, 0.610; chrysolaminaritol, $0 \cdot 563$.

It has been observed (Anderson et al. 1958) that oxidation of this type of polysaccharide under these conditions results in the stepwise removal of glucose and sorbitol units from the ends of the chains originally carrying reducing groups, through the formation of an intermediate malonaldehyde grouping, and 1 mole of formaldehyde is liberated from each glucose residue that is degraded. Mannitol residues linked through $\mathrm{C}_{(1)}$ or $\mathrm{C}_{(6)}$ and glucose units linked through $\mathrm{C}_{(6)}$ do not overoxidize, as no malonaldehyde grouping is formed. The presence of $40 \%$ of laminarin and laminaritol which is resistant to overoxidation is in agreement with the structure advanced by earlier workers.

In the absence of M-chains the incomplete oxidation of chrysolaminarin and its alcohol confirms the presence in the molecule of $1: 6^{\prime}$-linked glucose units or branch points (or both) which appear to occur on an average just over half way along the chains.

\section{DISCUSSION}

Results of these investigations agree with the earlier findings on laminarin that this polysaccharide consists essentially of chains of $\beta-1: 3^{\prime}$. linked glucose units and that some of the chains are terminated at the reducing end by mannitol residues. The results provide the first definite evidence for the presence of 2:3:4-tri-O-methylglucose in the methylated hydrolysate and hence confirm the presence of $1: 6^{\prime}$-linkages in a linear chain of $\beta-1: 3^{\prime}$-linked glucose residues. This is in accordance with the interpretation of Peat et al. (1958) from partial-hydrolysis studies on laminarin, and of Smith \& Unrau (1959) from their periodateoxidation and -reduction studies on this polysaccharide. Although the present experiments indicate that on an average $6 \%$ of the units in the linear chains are 1:6'-linked they provide no evidence for the relative position of the $1: 3^{\prime}$ - and $1: 6^{\prime}$-linkages in the molecule. The balance of evidence from previous work in these laboratories (Anderson et al. 1958; Hirst, O'Donnell \& Percival, 1958) was in favour of the occurrence of $1: 6^{\prime}$-linked glucose units only at branch points in the laminarin molecule and the present methylation studies suggest that on an average one branch point at $\mathrm{C}_{(\theta)}$ is present in each molecule. It follows that $1: 6^{\prime}$-linkages may be present in laminarin both in the linear chains and as interchain linkages. Further work is in progress to substantiate this. The proportion of tetra-O methylglucose agrees with the chain length of 16 glucose residues recorded by Barry (1942) from 
successive oxidation with periodate and bromine. The lower proportion of tetra- $O$-methylglucose reported by other workers was not from whole laminarin but from fractions of higher molecular weight.

It should be mentioned that the present results differ from those of Smith \& Unrau (1959) in that no evidence could be obtained for the presence of mannose in the sample of laminarin under investigation.

Chrysolaminarin, the polysaccharide synthesized by diatoms, has been shown to resemble laminarin in many respects. It consists of $\beta-1: 3^{\prime}-$ and $1: 6^{\prime}-$ linked glucose units in the ratio of 11:1 (cf. laminarin 15:1) with an average of one branch point at $\mathbf{C}_{(6)}$ in every molecule. However, the average chain length, calculated from the proportion of tetra-O-methylglucose present in the methylated hydrolysate, appears to be smaller in the chrysolaminarin: 12 glucose units instead of 16 units calculated for laminarin. Furthermore, whereas it was confirmed that a proportion of the laminarin molecules corresponding to $2 \%$ of mannitol are terminated at the reducing end by this sugar alcohol, no evidence for the presence of any unit other than glucose at the reducing end of the chrysolaminarin molecules could be obtained. This polysaccharide appears to differ from laminarin therefore in that it comprises only one of the types of molecule (G-chains) found in the latter.

From the formaldehyde liberated on oxidation with periodate both the chrysolaminarin and the sample of laminarin investigated appear to have a molecular weight of about 3500. This agrees with the figure reported by Broatch (1956) for methylated laminaritol.

\section{SUMMARY}

1. Crystalline chrysolaminarin separated from a mixture of diatoms (Chrysophyceae) had m.p. $273^{\circ},[\alpha]_{D}-6^{\circ}$ and was shown to contain $99.5 \%$ of glucose. Chromatographic examination of total hydrolysates of laminarin and of chrysolaminarin revealed the presence of mannitol in the former but not in the latter.

2. The alcohols obtained on reduction of the polysaccharides with borohydride gave on hydrolysis glucose, sorbitol and mannitol from laminarin and glucose and sorbitol from chrysolaminarin.

3. 2:3:4:6-Tetra-O-methylglucose, tri- $O$-methylglucose and di-O-methylglucose in the ratio of $1: 10 \cdot 5: 0.46$ and $1: 16: 0.6$ were present in the hydrolysates of methylated chrysolaminarin and laminarin respectively. The presence of the di-Omethyl sugar indicates the possible presence of one branch point per molecule.
4. The methylated sugars were separated on thick paper and evidence was obtained that both tri- $O$-methyl fractions consisted of a mixture of 2:4:6- and 2:3:4-tri- $O$-methylglucoses, in the ratio of about 11:1 and 15:1 in chrysolaminarin and laminarin respectively. This provides proof of the presence of $1: 6^{\prime}$-linkages in the linear $1: 3^{\prime}$-linked chains.

5. The amount of periodate reduced by both polysaccharides is in agreement with the presence of a high proportion of $1: 3^{\prime}$-linked glucose units.

6. The quantity of formaldehyde released by laminarin and chrysolaminarin and their respective alcohols on oxidation with periodate confirms the presence of mannitol-terminated chains (M-chains) in laminarin. No evidence of M-chains in chrysolaminarin could be obtained.

7. The degree of polymerization of both poly saccharides calculated from formaldehyde release is about 21 glucose units.

8. The incomplete oxidation of the two polysaccharides under conditions which facilitate degradation of 1:3'-linked glucose chains provides confirmation of the presence of $1: 6^{\prime}$-linked units in chrysolaminarin and of similar linkages or $\mathbf{M}$ chains or both in laminarin.

We are pleased to record our gratitude to Professor H. A. von Stosch for the supply of crystalline chrysolaminarin and are grateful to $\mathrm{Dr} \mathrm{W}$. C. Schaefer for the generous gift of 2:4-di-O-methylglucose. One of us (A.B.) is indebted to the Institute of Seaweed Research for a Maintenance Grant.

\section{REFERENCES}

Anderson, F. B., Hirst, E. L., Manners, D. J. \& Ross, A. G. (1958). J. chem. Soc. p. 3233.

Archibald, A. R., Manners, D. J. \& Ryley, J. F. (1958). Chem. \& Ind. p. 1516.

Aspinall, G. O. \& Ferrier, R. (1957). Chem. \& Ind. p. 1216.

Barry, V. C. (1942). J. chem. Soc. p. 578.

Bell, D. J. (1955). In Modern Methods of Plant Analysis, vol. 2, p. 9. Ed. by Paech, K. \& Tracey, M. V. Berlin: Springer Verlag.

Broatch, W. N. (1956). Ph.D. Thesis: University of Edinburgh.

Cifonelli, J. A. \& Smith, F. (1954). Analyt. Chem. 26, 1132.

Foster, A. B. (1952). Chem. \& Ind. p. 1050.

Hirst, E. L., O'Donnell, J. J. \& Percival, E. (1958). Chem. \& Ind. p. 834.

Hough, L. \& Perry, M. B. (1956). Chem. \& Ind. p. 768 .

Hough, L., Powell, D. B. \& Woods, B. M. (1956). J. chem. Soc. p. 4799.

Isbell, H. S., Frush, H. L., Bruchner, B. H., Kowkabany, G. G. \& Wampler, B. (1957). Analyt. Chem. 29, 1523.

MacFadyen, D. A., Watkins, H. D. \& Anderson, P. R. (1945). J. biol. Chem. 158, 107. 
Mackie, I. M. \& Percival, E. (1959). J. chem. Soc. p. 1151.

Parrish, F. W. (1959). Ph.D. Thesis: University of London.

Peat, S., Whelan, W. J. \& Lawley, H. G. (1958). J. chem. Soc. p. 729.

Perry, M. B. \& Jones, J. K. N. (1957). J. Amer. chem. Soc. 79, 2790.

Pringsheim, E. G. (1952). Quart. J. Sci. 93, 71.
Quillet, M. (1955). C.R. Acad. Sci., Paris, 240, 1001.

Schaefer, W. C. \& Van Cleve, J. W. (1956). Analyt. Chem. 28, 1290 .

Smith, F. \& Unrau, A. M. (1959). Chem. \& Ind. pp. 636, 881.

Somogyi, M. (1952). J. biol. Chem. 195, 19.

Stosch, H. A. von (1951). Naturwissenschaften, 38, 192.

\title{
The Effect of Succinate and Amytal on the Reduction of Acetoacetate in Animal Tissues
}

\author{
By H. A. KREBS, L. V. EGGLESTON AND A. D'ALESSANDRO \\ Medical Research Council Unit for Research in Cell Metabolism, Department of Biochemistry, \\ University of Oxford
}

(Received 14 November 1960)

It has recently been shown (Kulka, Krebs \& Eggleston, 1961) that succinate promotes the formation of $\beta$-hydroxybutyrate from acetoacetate in sheep-heart homogenates. An explanation was put forward which is based on the fact that succinate is oxidized in preference to substrates requiring a pyridine nucleotide-linked dehydrogenase. As the electrons from succinate and from reduced pyridine nucleotide travel to oxygen by a common pathway at the cytochrome level the preferential availability of the electrons from succinate blocks the transfer of electrons from reduced pyridine nucleotide to oxygen. At the same time the oxidation of succinate provides a substrate, malate, which reduces pyridine nucleotide. These two circumstances increase the amounts of reduced pyridine nucleotide available for the reduction of acetoacetate. This paper presents further evidence in support of this concept.

\section{METHODS}

Materials. Sodium acetoacetate and DL- $\beta$-hydroxybutyrate samples were as previously described. Crystalline L-lactic acid was prepared according to Krebs (1960), $\alpha$-oxoglutaric acid according to Friedman \& Kosower (1955); succinate-free crystalline monosodium oxoglutarate was prepared as follows. The crude acid was dissolved in 3 vol. of water and the solution was adjusted to $\mathrm{pH} \mathrm{3.7}$ with solid $\mathrm{Na}_{2} \mathrm{CO}_{3}, \mathrm{pH}$ being checked with a $\mathrm{pH}$ meter. The adjustment to this $\mathrm{pH}$ is critical as $\mathrm{p} K_{1}$ was found to be $2 \cdot 47, \mathrm{p} K_{2} 4 \cdot 68$ (determined electrometrically at $22^{\circ}$ ). After filtration 1 vol. of ethanol was added slowly and the resultant turbid solution was kept overnight in the refrigerator. The precipitate formed contained mostly impurities. A further volume of ethanol was added to the supernatant and after storage for $24 \mathrm{hr}$. at $0^{\circ}$ crystals of monosodium $\alpha$-oxoglutarate were collected. The purity was $96.5 \%$. A third volume of ethanol yielded material of $100 \%$ purity. This was used for the experiments. Amytal (5-ethyl-5isoamylbarbiturate) was added as the sodium salt.

Tissue preparations. Tissue homogenates were prepared and handled as previously described (Kulka et al. 1961). Any modifications in incubation time and tissue concentration are stated in the description of the experiments. In most cases the enzymic reactions were stopped by the addition of $1 \mathrm{ml}$. of $\mathrm{N}-\mathrm{HCl} / 4 \mathrm{ml}$. of homogenate. In other cases $\mathrm{HCl}$ was replaced by $15 \%$ perchloric acid.

Analytical methods. Acetoacetate was determined according to Edson (1935) or Walker (1954), citrate according to Taylor (1953), succinate according to Rodgers (1961), the sum of malate and fumarate according to Nossal (1952), pyruvate and $\alpha$-oxoglutarate according to Holzer \& Holldorf(1957), malate and lactate according to Hohorst, Kreutz \& Bücher (1959), glutamate according to Krebs \& Bellamy (1960), $\beta$-hydroxybutyrate according to

Table 1. Yield of ' $\beta$-hydroxybutyrate' of various compounds treated according to Greenberg \& Lester (1944)

The quantities tested correspond to maximum amounts present in the homogenates analysed.

$\begin{array}{lc}\begin{array}{c}\text { Substance tested } \\ (16 \mu \text { moles })\end{array} & \begin{array}{c} \\ \beta \text {-Hydroxybutyrate' } \\ \text { found } \\ (\mu \text { mole })\end{array} \\ \text { Succinate } & 0 \cdot 05 \\ \text { Fumarate } & 0 \\ \text { L-Malate } & 0 \\ \text { Citrate } & 0 \cdot 25 \\ \alpha \text {-Oxoglutarate } & 0 \cdot 16 \\ \text { Pyruvate } & 0 \cdot 36 \\ \text { L-Lactate } & 0 \cdot 05 \\ \text { Butyrate } & 0 \cdot 05 \\ \text { Crotonate } & 0 \cdot 14 \\ \text { Glucose } & 0.09 \\ \text { Amytal }(5 \mu \text { moles }) & 0.08\end{array}$

